\title{
Electron microscopical investigation of surface alterations on Giardia lamblia trophozoites after exposure to a cytotoxic monoclonal antibody
}

\author{
Received: 25 May 1995 / Accepted: 29 August 1995
}

\begin{abstract}
The present study describes a transmission electron microscopical investigation of trophozoites from Giardia lamblia clone GS/M-83-H7 after exposure to monoclonal antibody (MAb) G10/4. From previous studies it is known that this antibody immunoreacts with the parasite's major surface antigen VSP (variable surface protein) and exhibits a complement-independent cytotoxic effect on trophozoites of clone GS/M-83-H7. Our investigations revealed that cytotoxicity of MAb G10/4 is associated with shedding of VSP-containing membrane vesicles from the parasite surface and a concomitant partial disruption of the cellular membrane. These morphological alterations depend on the cross-linking capacity of the antibody because the immunoreactivity of respective monovalent $\mathrm{F}(\mathrm{ab})^{\prime}$ has no significant influence on the cell-surface structure. These findings indicate that the membrane-disintegrative activity of MAb G10/4 may be responsible for the parasitocidal function of the antibody.
\end{abstract}

\section{Introduction}

Giardia lamblia is a relevant causative agent of diarrhea in humans and various animals. In the last few years, this intestinal parasite has gained increasing importance as an infectious model organism for the investigation of intestinal immune reactions. Numerous immunocytochemistry and immunobiochemistry studies have revealed that the parasite undergoes continous antigenic variation that is mediated by the major surface antigen named VSP (variable surface protein; reviewed by Nash 1992).

The different variants of VSPs represent the primary targets for the local and systemic antibody response during a G. lamblia infection (Gottstein and Nash 1991; Gottstein et al. 1990, 1993). In particular, VSP-specific IgA antibodies have been demonstrated in the gut of $G$. lamblia-infected mice, and these antibody specificities

A. Hemphill · S. Stäger · B. Gottstein · N. Müller $(\bullet)$ Institute of Parsitology, University of Berne, P.O. Box 8466, CH-3001 Bern, Switzerland;

Fax: 41316312622 are supposed to be responsible for both the process of antigenic variation and the modulation of parasite infection (Gottstein et al. 1993). Due to their high antigenicity, VSPs also induce strong serum antibody responses in G. lamblia-infected individuals. Incubation of G. lamblia trophozoites with sera from infected humans (Hill et al. 1984) and rabbits (Nash et al. 1988) as well as with monoclonal antibodies (MAbs) to different variants of VSPs (Nash et al. 1988) has resulted in complement-dependent lysis of the parasites. In contrast, a MAb (6E7) to a $170-\mathrm{kDa}$ antigen (CRP 170) of G. lamblia clone WB (Nash and Aggarwal 1986) and a MAb (G10/4) to a 72$\mathrm{kDa}$ antigen (VSPH7) of clone GS/M-83-H7 (Aggarwal et al. 1989) have shown complement-independent cytotoxicity to the respective variant antigen types. As had been demonstrated in the case of MAb 6E7, high concentrations of such antibodies caused immediate immobilization and killing as well as aggregation of the trophozoites. At lower antibody concentrations, these specificities caused only aggregation but no cytotoxicity, whereas monovalent $\mathrm{F}(\mathrm{ab})^{\prime}$ of MAb 6E7 exhibited none of these cytological effects. These observations indicated that cytotoxicity was associated with but was not the ultimate consequence of antibody-mediated aggregation of trophozoites.

Since data reported by Nash and Aggarwal (1986) did not allow substantial conclusions to be drawn on the mechanism(s) of complement-independent, antibodymediated parasite killing, we performed an immunocytochemistry study that should give more insight into the cytological processes associated with this phenomenon. In particular, we investigated by transmission electron microscopy (TEM) the cell-surface alterations on trophozoites of $G$. lamblia clone GS/M-83-H7 that had previously been exposed to MAb G10/4. Incubation of viable cells with cytotoxic amounts of this antibody resulted in strong shedding of membrane vesicles and partial disrupture of the cellular surface. This finding indicates that the direct cytotoxic effect of MAb G10/4 may be due to a membrane-disintegrative process that destroys the integral structure of the parasite cells. 


\section{Materials and methods}

Origin and in vitro cultivation of Giardia lamblia trophozoites

Experimentation was performed with $G$. lamblia clone GS/M-83$\mathrm{H7}$, which originated from the human isolate GS and expresses a 72-kDa VSP (Aggarwal et al. 1989). Trophozoites from this clone were obtained by in vitro cultivation of cells at $37^{\circ} \mathrm{C}$ under anaerobic conditions in TYI-S-33 medium supplemented with antibiotics as described by Gottstein et al. (1990).

\section{Origin of MAbs and generation of $\mathrm{F}(\mathrm{ab})^{\prime} \mathrm{MAbs}$}

Ascites of MAb G10/4 reacting with the 72-kDa VSP (CSPH7) of clone GS/M-83-H7 (Aggarwal et al. 1989) as well as ascites of control MAb 6E7 reacting with the 170-kDa VSP on antigenically distinct clone WB (Nash and Aggarwal 1986) were kindly provided by T. Nash (NIH, Bethesda, Md., USA). Respective monovalent $\mathrm{F}(\mathrm{ab})$ 's were generated by papain digestion of MAbs according to Harlow and Lane (1988). Prior to their use, MAbs and $\mathrm{F}(\mathrm{ab})^{\prime} \mathrm{s}$ were heated for $30 \mathrm{~min}$ at $56^{\circ} \mathrm{C}$.

\section{Cytoxicity assay of antibody - or albendazole-treated parasites}

Trophozoites from a $15-\mathrm{ml}$ culture grown to the late logarithmic phase were rinsed once with $15 \mathrm{ml}$ phosphate-buffered saline (PBS, pH 7.4) at $37^{\circ} \mathrm{C}$ to remove nonviable and nonadheren cells. Adherent cells were dislodged by a 5-min incubation in 15 $\mathrm{ml}$ ice-cold PBS and were subsequently washed three times with ice-cold PBS by centrifugation at $800 \mathrm{~g}$ for $10 \mathrm{~min}$. After the final washing step, trophozoites were resuspended in TYI-S-33 medium at a density of $6 \times 10^{6}$ cells $/ \mathrm{ml}$. Then, $100-\mu 1$ aliquots of this suspension were distributed into wells of a round-bottomed microtiter plate (Corning, New York, N.Y., USA) and were then incubated for $20 \mathrm{~min}$ at $37^{\circ} \mathrm{C}$ in the absence or presence of MAbs at a dilution of 1:200. From each incubation, 10- $\mu \mathrm{l}$ aliquots corresponding to $6 \times 10^{4}$ trophozoites were subcultivated anaerobically for $24 \mathrm{~h}$ at $37^{\circ} \mathrm{C}$ in $15 \mathrm{ml}$ TYI-S-33 medium supplemented with antibiotics. Subsequently, adherent cells were dislodged and centrifuged as descibed above and then resuspended in $100 \mu \mathrm{l}$ ice-cold PBS. Finally, the cell numbers were determined microscopically from 10$\mu \mathrm{l}$ aliquots of each suspension using a Neubauer cell-counter chamber. All cytotoxicity assays were performed in triplicate.

Drug-mediated killing of $G$. lamblia trophozoites was performed according to Chavez et al. (1992) by incubation of $6 \times 10^{5}$ cells for $16 \mathrm{~h}$ at $37^{\circ} \mathrm{C}$ in $60 \mathrm{ml}$ TYI-S-33 medium containing antibiotics and albendazole (SmithKline-Beecham, Philadelphia, Pa., USA) at a final concentration of $1 \mu \mathrm{g} / \mathrm{ml}$. Then, dislodged cells were recovered from the culture medium by sedimentation, which was followed by two washes in ice-cold PBS and resuspension of the cells in $100 \mu \mathrm{l}$ of the same buffer. The efficiency of killing was tested using a $10-\mu \mathrm{l}$ aliquot $\left(5 \times 10^{4}\right.$ trophozoites) for a cytotoxicity assay as described above. The immobilization, detachment, and agglutination of antibody- or albendazole-treated trophozoites was assessed by repeated microscopical examination.

Cytochemical and immunocytochemical analyses of G. lamblia trophozoites

After incubation with antibodies or albendazole, respectively, trophozoites from the remaining $90-\mu$ l aliquots (see above) were washed three times in ice-cold PBS. They were fixed for $20 \mathrm{~min}$ in PBS containing 3\% paraformaldehyde and were washed again three times in PBS. Free aldehydes were quenched by incubation of the preparation in PBS containing $50 \mathrm{~m} M$ glycine for $1 \mathrm{~h}$ at room temperature, and blocking of unspecific binding sites was done by resuspension of the cells in PBS/0.5\% BSA/50 $\mathrm{m} M$ glycine for $30 \mathrm{~min}$ at room temperature. The cells were then washed once in PBS and then incubated for $1 \mathrm{~h}$ with a goat anti-mouse antibody conjugated to 10 -nm gold particles (Amersham, Zürich, Switzerland) diluted 1:5 in blocking solution. Immediately before use, the gold-conjugates were centrifuged at $4800 \mathrm{~g}$ for $10 \mathrm{~min}$ to remove possible aggregates (Hemphill et al. 1991). The labeled parasites were washed three times for $5 \mathrm{~min}$ in PBS and were subsequently fixed and processed for TEM (see below).

Alternatively, G. lamblia trophozoites were fixed in PBS containing 3\% paraformaldehyde for $20 \mathrm{~min}$ at room temperature prior to the MAb treatments. Trophozoites were then washed three times in PBS before the blocking of free aldehydes as described above. Incubations with MAbs were carried out at a dilution of 1:200 in TYI-S-33 medium as described for live trophozoites (see above). After being washed in PBS, the parasites were incubated with the gold-conjugated goat anti-mouse antibody and were finally washed three times for $5 \mathrm{~min}$ in PBS prior to processing for TEM.

Immunogold-labeled and albendazole-treated trophozoites were fixed in $100 \mathrm{~m} M$ sodium phosphate buffer containing 3\% glutaraldehyde for $3 \mathrm{~h}$ at $4^{\circ} \mathrm{C}$, were washed several times in PBS, and were postfixed in $2 \%$ osmium tetroxide in phosphate buffer for $3 \mathrm{~h}$ at $4^{\circ} \mathrm{C}$. Parasites were then washed several times in $\mathrm{H}_{2} \mathrm{O}$ and were incubated in $1 \%$ uranyl acetate in $\mathrm{H}_{2} \mathrm{O}$ for $1 \mathrm{~h}$. The specimens were dehydrated through a serial ethanol gradient $(50 \% / 70 \% / 90 \% / 100 \%)$ and were finally embedded in Epon 812 resin (Fluka, Buchs, Switzerland). Polmyerization of the resin was achieved at $65^{\circ} \mathrm{C}$ over a period of $24 \mathrm{~h}$.

Sections for TEM were cut on a Reichert \& Jung ultramicrotome using a Diatome diamond knife and were loaded onto 200mesh nickel or copper grids. They were stained with uranyl acetate and lead citrate (Smith and Croft 1991), and specimens were viewed and photographed using a Phillips 600 transmission electron microscope operating at $60 \mathrm{kV}$.

\section{Results}

The strong cytotoxic effect of MAb G10/4 on in vitrocultivated trophozoites of Giardia lamblia clone GS/M83-H7 is illustrated in Fig. 1. It is shown that as com-

living cells $\times 10^{4}$ (after subcultivation)

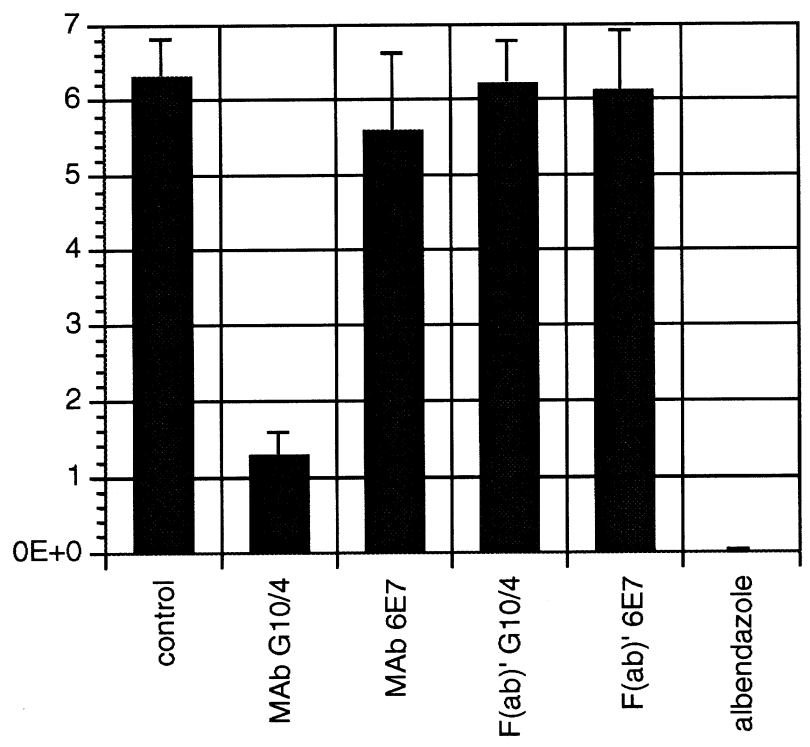

Fig. 1 Cytotoxicity assays of Giardia lamblia trophozoites. In all, $6 \times 10^{4}$ trophozoites from G. lamblia clone GS/M-83-H7 either left without treatment (control) or treated with MAbs, respective $\mathrm{F}(\mathrm{ab})^{\prime} \mathrm{s}$, or albendazole were subcultured in fresh medium under optimal growth conditions. The data represent the total number of surviving parasites after subcultivation. Note that cytotoxicity was observed after MAb G10/4 and albendazole treatment, whereas MAb 6E7 and the F(ab)'s of the two MAbs used did not exhibit any cytotoxicity at all 

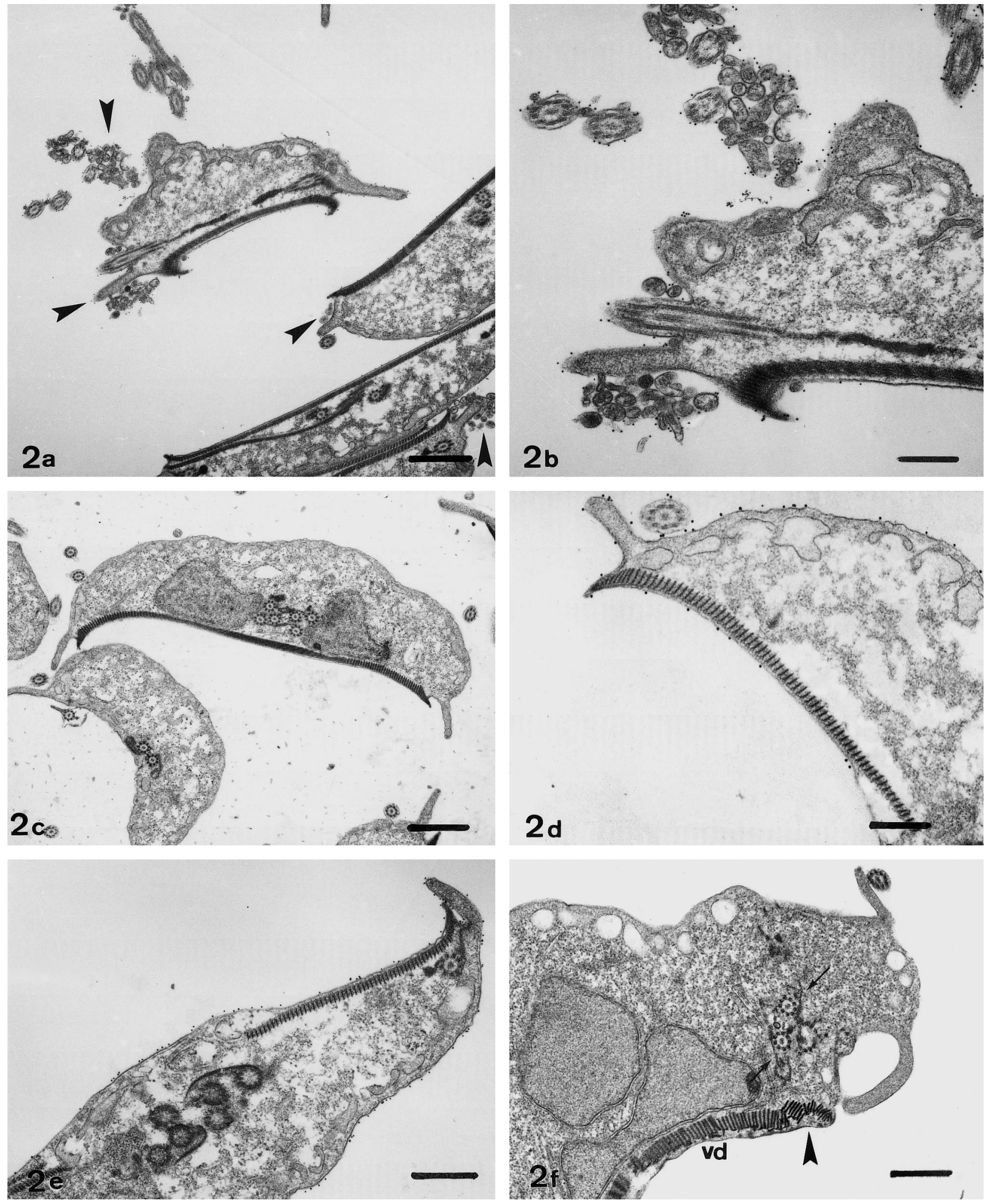

Fig. 2a-f TEM of G. lamblia trophozoites. a, b Live parasites were incubated in the presence of cytotoxic MAb G10/4, fixed briefly in $3 \%$ paraformaldehyde, and labeled with an anti-mouse antibody conjugated to 10-nm gold particles. Note the extensive shedding of VSP-containing vesicles from the parasite surface (arrowheads). a Bar $=990 \mathrm{~nm}$. b Bar $=400 \mathrm{~nm}$. c-e Trophozoites were incubated in the presence of MAb6E7 (c bar=1100 nm) or in the presence of $\mathrm{F}(\mathrm{ab})^{\prime} \mathrm{s}$ of MAbG10/4 (d bar=400 nm) or parasites were prefixed

with $3 \%$ paraformaldehyde prior to immunogold labeling with MAbG10/4 (e bar=450 nm). Note the complete absence of cell-surface alterations upon these treatments. $f$ Parasites were treated with albendazole prior to fixation and processing for TEM. Note the typical alterations upon albendazole treatment, namely, distortion of the ultrastructure of the ventral disc ( $v d$, arrowhead) and abnormal microtubule organization within the cell (arrows). No vesicle formation on the surface of the trophozoites was observed. Bar=660 nm 
pared with the control experiment performed with MAb $6 \mathrm{E} 7$, exposure of parasite cells to MAb G10/4 resulted in a strongly reduced recovery of viable cells upon a 24-h subcultivation carried out under optimal growth conditions. MAb G10/4 treatment went along with an immediate immobilization and detachment of trophozoites, followed by a strong agglutination of the dislodged parasites occurring at about $5 \mathrm{~min}$ after antibody addition. In contrast, incubation of trophozoites with control MAb 6E7, with MAb G10/4-derived F(ab)', or with control $\mathrm{F}(\mathrm{ab})^{\prime}$ did not generate any of these effects.

Immunogold TEM exhibited remarkable morphological changes on the surface of MAb G10/4-treated trophozoites. Careful analysis of thin sections clearly revealed strong membrane vesicle formation as a consequence of the reaction of $\mathrm{MAb}$ G10/4 with the parasite surface (Fig. 2a). As can be seen in more detail in Fig. 2b, some of the cells were heavily deformed and exhibited extensive cell-surface alteration. Vesicles were $50-200 \mathrm{~nm}$ in diameter and were strongly labeled with immunogold particles, indicating a high content of VSP. The respective preparations also contained a few trophozoites that had undergone antigenic switching during previous in vitro cultivation and, thus, did not react with MAb G10/4 (not shown). On these cells, no significant change in surface topography could be observed. Also, shedding of vesicles was not visible on cellular surfaces of trophozoites that had previously been treated with monovalent F(ab)' MAb G10/4 (Fig. 2d) or with control MAb 6E7 (Fig. 2c) or that had been fixed with formaldehyde prior to exposure to the antibody (Fig. 2e). In summary, these findings indicate that cytotoxicity and aggregation as well as vesicle formation on the surface of $G$. lamblia trophozoites depend on the cross-linking function of MAb G10/4.

To test whether the above-described morphological appearance was due to the action of MAb G10/4 or whether it was a general phenomenon associated with cell death, trophozoites were killed by treatment with the parasitocidal drug albendazole (see Fig. 1) and subsequently analyzed by TEM. In aggreement with the observations of Chavez et al. (1992), albendazole treatment resulted in strong modifications of the cell shape associated with significant deformation of the adhesive disks and distortion of the microtubular organization (Fig. 2f). However, in terms of vesicle formation, this investigation demonstrated no striking morphological difference between treated and untreated cells. Thus, the observed antibody-mediated change in cell-surface morphology is strictly associated with the cytotoxic function of MAb G10/4 and does not seem to be a consequence of cell death per se.

\section{Discussion}

In the present study we investigated morphological alterations on the surface of trophozoites from Giardia lamblia clone GS/M-83-H7 that were triggered by MAb
G10/4. Immunocytochemical TEM investigations of trophozoites treated with this MAb demonstrated abundant surface membrane vesicle formation that seemed to be associated with disintegration or weakening of the cellular membrane. Morphological changes were not visible on the surface of trophozoites that had previously been exposed to monovalent F(ab)' MAb G10/4. These observations suggested that the formation of membrane vesicles relied on the potential of the antibody to cross-link neighboring VSP molecules. This process is likely to be directly responsible for the antibody-mediated cytotoxicity and is not only a symptom of cell death per se. This conclusion was confirmed by the finding that killing of the parasites through the action of albendazole was not associated with membrane vesicle formation at all.

The data presented in this paper describe the cytotoxic effect of MAb G10/4 as an efficient process of parasite killing. This finding emphasizes the questions raised in earlier experiments (Gottstein and Nash 1991; Gottstein et al. 1990,1993$)$ as to whether such cytotoxic antibody specificities play a physiological role in modulating antigenic variation and whether they are involved in the process of local parasite clearance during a G. lamblia infection. Evidence for a possible function of antibody-mediated parasitocidal mechanisms in controlling G. lamblia infections had previously been obtained by investigation of the local antibody response in infected mice. Results of model G. muris and G. lamblia infections in mice have suggested that parasite-specific intestinal secretory IgA (Anders et al. 1982; Snider and Undertown 1986; Sharma and Mayrhofer 1988) and, in particular, anti-VSP IgA (Gottstein et al. 1993) may be essential for an influence on the course of infection. In this context, it is conceivable that intestinal anti-VSP IgA specificities display the potential of a direct cytotoxic effect on $G$. lamblia trophozoites and, thus, may be responsible for, or may contribute to, the immunological effector mechanisms that are involved in intestinal parasite clearance. A study investigating this hypothesis is currently in progress.

Acknowledgements We would like to thank Verena Zimmermann and Toni Wyler for their excellent technical assistance and Ted Nash for providing us with G. lamblia clone GS/M-83-H7 and MAb G10/4. This study was supported by a grant obtained from the Swiss National Science Foundation (number 31-37607.93).

\section{References}

Aggarwal A, Merritt JW, Nash TE (1989) Cysteine-rich variant surface proteins of Giardia lamblia. Mol Biochem Parasitol 32:39-48

Anders RF, Robert-Thomson IC, Mitchell GF (1982) Giardiasis in mice: analysis of humoral and cellular immune response to $\mathrm{Gi}$ ardia muris. Parasite Immunol 4:47-57

Chavez B, Cedillo-Rivera R, Martinez-Palomo A (1992) Giardia lamblia: ultrastructural study of the in vitro effect of benzimidazoles. J Protozool 39:510-515

Gottstein B, Nash TE (1991) Antigenic variation in Giardia lamblia: infection of congenitally athymic nude and scid mice. Parasite Immunol 13:649-659 
Gottstein B, Harriman GR, Conrad JT, Nash TW (1990) Antigenic variation in Giardia lamblia: cellular and humoral immune response in a mouse model. Parasite Immunol 12:659-673

Gottstein B, Deplazes P, Tanner I (1993) In vitro synthesized immunoglobulin A from nu/+ and reconstituted nu/nu mice against a dominant surface antigen of Giardia lamblia. Parasitol Res 79:644-648

Harlow E, Lane D (1988) Antibodies: a laboratory manual. Cold Spring Harbor Laboratory Press, Cold Spring Harbor, New York

Hemphill A, Seebeck T, Lawson D (1991) The Trypanosoma brucei cytoskeleton: ultrastructure and localization of microtubule-associated and spectrin-like proteins using quick-freeze, deep-etch, immunogold electron microscopy. J Struct Biol 107:211-220

Hill DR, Burge JJ, Pearson RD (1984) Susceptibility of Giardia lamblia trophozoites to the lethal effect of human serum. J Immunol 132:2046-2052
Nash TE (1992) Surface antigen variability and variation in Giardia lamblia. Parasitol Today 8:229-234

Nash TE, Aggarwal A (1986) Cytotoxicity of monoclonal antibodies to a subset of Giardia isolates. J Immunol 136:26282632

Nash TE, Aggarwal A, Adam RD, Conrad JT, Merritt JW (1988) Antigenic variation in Giardia lamblia. J Immunol 141:636641

Sharma AW, Mayrhofer G (1988) A comparative study of infections with rodent isolates of Giardia duodenalis in inbred strains of rats and mice in hypothymic nude rats. Parasite Immunol 10:169-179

Smith M, Croft SL (1991) Embedding and thin section preparation. In: Rickwood D, Harris BD (eds) Electron microscopy in biology. A practical approach. IRL Press, Oxford, pp 17-37

Snider DP, Underdown BJ (1986) Quantitative and temporal analyses of murine antibody response in serum and gut secretions to infection with Giardia muris. Infect Immun 52:271-278 